Revue d'histoire de l'Amérique française

REVUE D.HISTOIRE DE L'AMÉRIQUE FRANÇAISE

\title{
Le comportement du clergé pendant les opérations militaires de la conquête
}

\section{Marcel Trudel}

Volume 7, numéro 3, décembre 1953

URI : https://id.erudit.org/iderudit/301605ar

DOI : https://doi.org/10.7202/301605ar

Aller au sommaire du numéro

Éditeur(s)

Institut d'histoire de l'Amérique française

ISSN

0035-2357 (imprimé)

1492-1383 (numérique)

Découvrir la revue

Citer cet article

Trudel, M. (1953). Le comportement du clergé pendant les opérations militaires de la conquête. Revue d'histoire de l'Amérique française, 7(3), 314-340.

https://doi.org/10.7202/301605ar
Résumé de l'article

Le port des armes, interdit aux gens d'Eglise. Quelques aumôniers célèbres. La brève captivité d'un jésuite. Des religieux accusés d'activité militaire. Les directives de l'Évêque et du Gouverneur. Trois amateurs en défense militaire. Le belliqueux Parent perd sa cure. On emprisonne Youville-Dufrost, puis on l'exile. D'autres arrestations ? Le curé Portneuf, victime de sa stratégie. L'ecclésiastique Couillard, tué dans une escarmouche. Conclusion. 


\title{
LE COMPORTEMENT DU CLERGÉ PENDANT LES OPÉRATIONS MILITAIRES DE LA CONQUETE*
}

\begin{abstract}
Sommaire
Le port des armes, interdit aux gens d'Eglise. Quelques aumôniers célèbres. La brève captivité d'un jessuite. Des religieux accusés d'activite militaire. Les directives de l'Évêque et du Gouverneur. Trois amateurs en défense militaire. Lo belliqueux Parent perd sa cure. On emprisonne Youville Dufrost, puis on l'exile. D'autres arrestations? Lo curé Portneuf, victime de sa stratégie. L'ecclésiastique Couillard, tue dans une escarmouche. Conclusion.
\end{abstract}

Comment le clergé de la vallée du Saint-Laurent va-t-il se comporter pendant les opérations de la conquête? Chefs spirituels et, très souvent, conseillers temporels des Canadiens, les prêtres ne seraient-ils pas tentés d'intervenir personnellement dans le conflit?

Le PORT des ARMes, INTERdit AUX Gens D'ÉgLISE

Assurément le clergé n'avait pas le droit de prendre les armes et, comme l'indique le Rituel du diocèse de Québec ${ }^{1}$, le port des armes, "dans une guerre même juste", rendait inapte à recevoir les ordres sacrés; c'est pourquoi lorsqu'un jeune Canadien, Pierre Mennard, débarque en France au mois de décembre 1760 pour s'y préparer aux ordres, il doit au préalable obtenir de Rome une absolution a gestatione armorum qui lui coâte 110 livres $^{2}$. Dès l'ouverture de la

* Extrait d'un ouvrage en préparation, L'Église canadienne sous le régime militaire.

1. Rituel du diocèse de Québec (éd. 1703), 326.

2. Voir Marcel Trudel, Note supplémentaire sur messire Pierre Mennard, Canadien, dans RHAF, VII, 2 (septembre 1953): 290-292. 
campagne, l'évêque prit soin de donner à son clergé des dírectives précises: "Si par hasard l'ennemi descendait à une paroisse et s'en rendait le maitre, le curé lui fera toutes les politesses possibles; le priera d'épargner le sang et les églises. In ne sera point armé, non plus que les aumôniers des camps"; et il ajoute, au cas où des prêtres, par la force des circonstances, se feraient soldats: "tous les confesseurs déjà approuvés dans tout le diocèse... pourront dispenser de l'irrégularité, dans le tribunal, les prêtres qui l'auraient encourue par l'homicide volontaire de l'ennemil, ad sui vel patriae defensionem ${ }^{3}$."

Les prêtres pouvaient accompagner l'armée, à titre d'aumôniers militaires, comme ils l'avaient toujours fait depuis les débuts de la colonie et l'on sait que, depuis 1692 , les Récollets étaient les aumôniers militaires attitrés'. Ces aumôniers, ainsi qu'on vient de le voir dans la lettre épiscopale, n'avaient pas le droit de porter les armes; par une convention internationale, ils se trouvent sur le pied des non-combattants: les aumôniers "ne pourront être faits prisonniers"s.

\section{QUelques AUMôNIERS CÉLÈBRES}

Nous connaissons quelques-uns de ces aumôniers qui ont servi pendant les opérations de la conquête. Parmi les cinq récollets de Québec que le Commissaire, à la demande de l'évêque, destine à l'aumônerie de l'armée en juin 1759 , nous pouvons identifier le P. Gabriel Anheiser ${ }^{6}$, à qui il arrivera bientôt de perdre tous ses livres, lors d'une descente des Anglais à Deschambault?. Les récollets Félix Berey, Antoine Deperet et Bonaventure Carpentier

3. Lettre circulaire de Mgr de Pontbriand, 5 juin 1759, Mandements, III: 137-139.

4. Edits, ordonnances royaux, I: 275 (édit de mars 1692).

5. Cartel conclu entre la France et l'Angleterre, le 6 février 1759, dans Knox, Historical Journal, III: 272.

6. Journal de Foligné, dans Doughty, Siege of Quebec, IV: 169. Il signe Gabriel Anheiser à l'Hôpital-Général de Québec, jusqu'au 28 mars 1759: AHG-Q, Décès, $1728-1783$.

7. Les Anglais incendièrent le dépôt où l'on avait réuni les équipages des troupes: "cette incendie cout au pere Gabriel une malle qui le deranga beaucoup pr. un Recollet, il y avoit tous ses livres" (ibid., 197). 
sont sur le lac Champlain ou dans les forts du Richelieu; les récollets Etienne Cotton et Ambroise Rouillard servent d'aumôniers militaires à Ristigouche'; le P. Virot, jésuite, se fait tuer par les Iroquois, le 24 juillet 1759 , lors d'une sortie des troupes françaises dans les environs du fort Niagara ${ }^{10}$; le jésuite Le Bansais, de Québec, fait occasionnellement du ministère auprès de l'armée où il devient d'ailleurs la cible du malicieux Montcalm ${ }^{11}$. Des aumôniers accompagnent aussi les sauvages sur le sentier de la guerre: les sulpiciens François Picquet, Jean-Claude Mathevet et François-Auguste Magon de Terlaye ${ }^{12}$; le jésuite Germain monte d'Acadie avec les sauvages de sa mission ${ }^{13}$; le jésuite de la Brosse est à la Pointeaux-Trembles avec ses Indiens ${ }^{14}$; le jésuite Roubaud, à la Canardière, accompagne ses Abénaquis de Saint-François-du-Lac ${ }^{15}$.

Pour avoir droit à l'immunité que leur conférait le cartel de février 1759 , ces aumôniers se sont-ils limités à leur seul rôle spirituel ? Il appert par les documents que certains d'entre eux ne s'en tenaient pas toujours à leur ministère auprès des âmes. Quand le P. Germain arrive à Kamouraska avec ses sauvages d'Acadie, le

8. APQ, Registres des Forts Saint-Jean, Duquesne, Chateauguay, Beauharnois (F. Fréderic) 17\$2-1760; P.-G. Roy, Hommes et choses du fort Saint-Frederic; A. Godbout, Les aumoniers du Richelieu, dans Soc. canad. hist. de l'Eglise, 1945-1946, $\mathbf{7 4}, \mathbf{7 7}, \mathbf{7 9 s}$. Nous trouvons Félix Berey au fort St-Jean et au fort Saint-Frédéric en $\mathrm{j}^{\mathrm{anvier}} \mathbf{1 7 6 0}$. Le P. Deperet (c'est ainsi qu'il signe) est au fort St-Jean en mai 1759.

9. APQ, Registres des Baptêmes Mariages et Sepultures de la paroisse ste anne de Ristigouche. Le P. Rouillard s'intitule "aumônier du Machaud" et le P. Cotton, aumônier des troupes de la Marine.

10. RJ, LXXI: 178.

11. Il signe Simeon le Bansais dans les registres de Notre-Dame-de-Québec, le 2 novembre 1759. Montcalm, qui ne ménageait personne, s'est bien moqué du P. Le Bansais; à propos de 200 Canadiens qui partent en canots en juin 1759, il écrit: "Il n'y avoit nul officier pour leur parler, et ils étoient réduits aux exhortations du $P$. Beausset, jésuite, qui les tournoit à la manière de celles qu'on fait aux gens qu'on veut pendre" (Coll. Levis, VII: 547s). Une autre fois, il écrit: "Les remparts de Rebbais (?) au lieu des sermons du P. Beausset" (ibid., VII: 552). Un sergent déserteur se présente-t-il: "Le P. Beausset, écrit Montcalm, lui a sauté au collet à son arrivée et l'a prié de se confesser, ce qu'il a refusé" (ibid., VII: 598).

12. Voir O. Maurault, "Quand Saint-Sulpice allait en guerre," dans les Cahiers des Dix, V (1940): 22-29.

13. Mémoire de Vaudreuil à Boishébert, 5 mars 1759, ibid., VI: 130.

14. APQ, Journal de M. Jean-Félix Récher, III: $47 \mathrm{~s}$.

15. Roubaud à Bougainville, 11 septembre 1759, dans Doughty, Siege of Quebec, IV: 122. 
gouverneur Vaudreuil enjoint au sieur de Boishébert de concerter "avec ce Missionnaire, le parti qui sera le plus co:tvenable à leur égard" et il ajoute cette phrase importante: "Nous sommes bien persuadés que Mrs. les Missionnaires qui sont auprès de ces accadiens et sauvages concourront chacun de leur mieux à l'exécution de nos intentions ${ }^{16}$ ". Le chevalier de La Corne compte sur le récollet Berey pour obtenir des renseignements: "J'ai toujours attendu le P. Berey qui est à Frontenac, pour savoir ce qui se passe chez les Anglois et s'ils doivent venir icir". Le jésuite Roubaud, posté à la Canardière, regrette de n'avoir pas réussi à mettre ses sauvages au service de l'expédition que projette Bougainville et il écrit: "J'ai rendu compte a $M$. le Marquis de Montcalm de tous les mouvements que vous vous donniez pour pourvoir à tout, il m'a paru reconnoitre son élève dans vos manœuvres... Rien de nouveau ici. L'ennemi est venu ce matin sonder le bassin de Quebeck. c'est u. e montre et rien de plusis." Détails peu importants, nous le concédons, mais si ces documents étaient tombés entre les mains des Anglais, ils auraient pu s'en prévaloir pour priver ces aumô:: iers de l'immur. ité.

Chez les Sulpiciens, on connait Magon de Terlaye qui, à cause de son humeur guerrière, a été surnommé par Bougainville chevalier Terlaye ${ }^{19}$. Mais c'est surtout le sulpicien François Picquet qui s'est rendu célèbre pour son rôle militaire auprès des sauvages. Doué d'un grand prestige auprès des Iroquois catholiques, il s'était appliqué, en fondant la Présentation en 1749, à soustraire de l'influence anglaise le plus possible d'Iroquois, programme politique et militaire autant que programme d'évangélisation. Dars la campagne de 1759, Picquet rend de grands services par l'autorité qu'il exerce sur ses sauvages. La Corne écrit de l'Ile-aux-Galops en août: "Nos sauvages sont dans les meilleurs sentiments du monde et disent qu'ils n'abandonneront jamais le François. M. Piquet est toujours des plus zélés et les conduit on ne peut mieux ${ }^{20 "}$. Il était tellement

16. Mémoire de Vaudreuil à Boishébert, 5 mars 1759, dans Doughty, op. cit., VI: 130 .

17. La Corne à Lévis, 10 août 1759, Coll. Lévis, X: 201.

18. Roubaud a Bougainville, 11 septembre 1759, dans Doughty, op. cit., IV: 122

19. Maurault, op. cit., dans les Cahiers des Dix, V (1940): 29. Il serait demeuré a la Présentation jusqu'en 1759 (Gauthier, Sulpitiana, 230).

2 La Corne à Lévis, 17 aout 1759, Coll. Lévis, X: 206. 
zélé que peu de jours auparavant, avec LaCorne, il s'était mis à la poursuite de six sauvages partis faire leur paix avec les Anglais ${ }^{21}$. Bref, le sulpicien Picquet, comme le chevalier de Beauclair l'écrit à Lévis, "a fait sa campagne en guerrier22". La tête de Picquet avait été mise à prix dès $1756^{23}$; il n'est pas sûr qu'elle le fût encore en 175924: cependant, voyant que la cause était perdue en 1760 et refusant de se soumettre à l'allégeance anglaise, Picquet quitta Montréal en septembre et prit la route de la Nouvelle-Orléans ${ }^{25}$.

\section{LA BRÈve CAPTIVITÉ D'UN JÉSUITE}

Parmi ces aumôniers qui accompagnaient les combattants, y en eut-il qui furent faits prisonniers? Nous pouvons en identifier un d'une façon certaine: le jésuite de la Brosse. Occupé à faire du ministère àYamaska où il signe les registres jusqu'au 6 juin $1759^{26}$, le Père de la Brosse quitte peu après sa paroisse et nous le trouvons en juillet suivant avec des sauvages à la Pointe-aux-Trembles (Neuville). Or, le 21 juillet, les Anglais font à cet endroit une descente-éclair: ils parcourent les maisons et capturent "environ 200 femmes dont la majeure partie étoit venue de Québec y chercher une retraite"; le Père de la Brosse est capturé et mis à bord d'un navire $^{27}$, et avec lui une religieuse de la Congrégation, la jeune Sœur

21. Le même au même, 10 aout 1759, ibid., X: 203.

22. Beauclair à Lévis, 23 octobre 1759, ibid., X: 189.

23. Journal de Montcalm, 10 novembre 1756, ibid., VII: 119.

24. Amherst ne lui aurait pas gardé rancune: Chagny, François Picquet, "le Canadien", 585s.

25. Arrivé à la Nouvelle-Orléans au cours de l'été 1761, Picquet y demeure jusqu'en avril 1763, après quoi il rentre en France. Sur Picquet, voir Chagny, op. cit., 583ss; Gosselin, Le fondateur de la Présentation (Ogdenbsurg): l'abbé Picquet, dans MSRC, XII (1894), 3-28; Maurault, op. cit., dans les Cahiers des Dix, V (1940): 22ss.

26. Yamaska, registre des baptêmes, mariages et sépultures, I (1727-1775). Il signe de la Brosse.

27. Relation du siège de Québec, dans Mémoire du Sieur de Ramezay, 12; APQ, Journal de M. Jean-Félix Récher, III: 47s. Le curé Récher est le seul à identifier le P. de la Brosse; ailleurs, on parle d'un jésuite sans le nommer: Journal of the Particular Transactions, dans Doughty, Siege of Quebec, V: 173; The Townshend Papers, ibid., V: 250; journal de Wolfe, dans Knox, Historical Journal, I: 439, n. 1. 
Sainte-Agnès, née Elisabeth Parent, et ses huit pensionnaires ${ }^{28}$. Le commandant anglais traita dignement le jésuite, il le laissa confesser les deux cents prisonnières et lui déclara même "qu'il pouvait dire la messe, s'il le voulait". La captivité du Père de la Brosse fut de très courte durée: dès le lendemain, il fut remis en liberté avec ses prisonnières ${ }^{29}$ et nous le retrouvors presque tout de suite à Yamaska, où il signe un registre dès le 6 août suivant: il avait terminé sa carrière d'aumônier. Un autre aumônier (du moins, nous croyons qu'il s'agit d'un aumônier) que nous re pouvons identifier, fut capturé à Québec le 16 septembre 1759 , en même temps qu'une trentaine de Canadiens ${ }^{30}$ : c'est tout ce que nous pouvons savoir de lui.

\section{Des Religieux aCCUSÉS D'aCtivité militaire}

A l'article des aumôniers, rattachons immédiatement les religieux que les documents de l'époque mêlent aux opérations de la conquête. Nous sommes ici en présence de témoignages pas toujours sûrs, qui n'identifient pas toujours clairement les religieux en cause. Lorsque des officiers anglais, détachés de l'armée du lac Champlain, se font prendre par des Abénaquis de Saint-François, Montcalm écrit dans son journal en août 1759: "Le P. Riverain, jésuite, a envoyé chercher du monde aux Trois-Rivières, qui a conduit le tout à bord de M. Canon": qu'il s'agisse là du scolastique Rivalin ou du P. Roubaud ${ }^{31}$, il n'entrait aucunement dans son rôle de s'occuper de cette affaire. En octobre 1759, nous trouvons les Jésuites mêlés à une razzia d'animaux sur les terrains de l'Hôpital Général de Québec. La cavalerie française y ayant enlevé un grand nombre de bêtes, écrit l'annaliste de l'Hôpital, le général anglais "s'avisa sur le champ d'un moyen aussi étranger et singulier qu'il paraît avoir été efficace: ce fut d'envoyer en toute hâte un exprès chez le

28. Voir notre chapitre sur la Congrégation de Notre-Dame. La Sœur SteAgnès n'avait alors que 26 ans.

29. Journal de Wolfe, dans Knox, op. cit., I: 439s., n. 1.

30. Knox, Historical Journal, II: 119.

31. Journal de Montcalm, Coll. Lévis, VII: 596. On ne connait point de $\mathbf{P}$. Riverain; Montcalm a peut-être confondu avec le scolastique Rivalin (voir RJ, LXXI: 180) ou bien c'est le copiste qui a mal lu: en ce cas, il s'agirait très probablement du P. Roubaud. 
supérieur des jésuites, avertissant ce dernier que, si les animaux enlevés à l'Hôpital Général n'étaient pas rapportés dans un certain espace de temps, les pères de la compagnie seraient bannis de la capitale, et ne pourraient plus y reparaître sous peine de mort. Les rusés jésuites fire:at savoir le tout au commandant des troupes françaises et justice était faite à qui de droit, même avant la fin du jour ${ }^{32}$." Rien n'établit la participation des Jésuites à cette razzia: le général anglais a peut-être simplement voulu faire du chantage ou bien Knox, qui est le premier à rapporter cette histoire, s'est fié trop facilement à des commérages. En novembre, toujours selon Knox, on aurait trouvé caché par le valet d'un jésuite "un baril de poudre, avec un tonreau de 15,000 cartouches" et Knox ajoute: ce jésuite "a insirué que son domestique est un imbécile et il espère par conséquent que tout témoignage de ce valet sera écarté; ce à quoi il lui fut répondu: que si cette poudre avait été cachée sous terre quatre ou six semaires auparavant, elle aurait été endommagée par la température extrêmement humide que nous avions à cette époque; mais comme cette température a été suivie d'une forte gelée, cela démontre clairement que si le garçon est un imbécile, ses instigateurs et ses complices ne sont pas des fous". C'est la réponse que Murray aurait faite à ce jésuite, mais Knox n'en est pas tout à fait sûr $^{33}$ et nous n'avons point d'autres précisions sur cette affaire de munitions cachées sous terre par un jésuite après la capitulation de Québec.

Quel est ce religieux (est-ce un jésuite ou un récollet ?) que l'on soupçonne en novembre 1759 d'avoir engagé des soldats anglais à déserter? Knox parle d'un vieux religieux qu'il eut ordre d'arrêter: "Je le trouvai chez lui et l'arrêtai au nom de Sa Majesté Britannique; le pauvre vieillard fut grandement terrifié et me supplia instamment de lui dire pour quel crime, mais je ne tardai pas davantage à poster une garde qui m'accompagnait, dans la chambre de ce vieux Père." L'enquête terminée et aucune preuve n'ayant été établie, le religieux fut remis en libertét ${ }^{34}$.

32. L'Hôpital Général, 355s. Le récit de l'annaliste est tiré de Knox, Historical Journal, II: 242s., y compris les mots crafty fathers employés par Knox.

33. Knox, Historical Journal, II: 274.

34. Ibid., II: 278 et 280 . Il restait chez les Jésuites deux religieux très âgés: le $P$. Richer, qui avait 77 ans, et le P. Lauverjat, qui en avait 80; Knox parle d'un 
Un mémoire, préparé au cours de l'hiver 1759-1760, sur les moyens de reprendre la capitale, propose que l'on recoure aux Jésuites: "Pour se procurer des espions affidés, on pourroit s'adresser aux Jésuites. Ils sont propres à inspirer le zèle nécessaire pour risquer sa vie dans une besogne où peut entrer le motif de la religion ${ }^{35}$." A-t-on mis ce projet à exécution? Pour sa part, le jésuite Gounon, missionnaire à Bécancour, envoie à Vaudreuil, le 17 avril 1760, des renseignements qu'il a reçus de Québec, mais ces renseignements, selon Vaudreuil, exagéraient "la maladie et la foiblesse de la garnison angloise" 36 . Enfin, en juin 1760, Vaudreuil annonce que "quelques Jesuites" sont détenus prisonniers à bord d'un vaisseau de guerre ${ }^{37}$ : ce sont peut-être ces mêmes jésuites que l'on a arrêtés, selon Knox, pour avoir excité les Canadiens à la révolte ${ }^{38}$; en juillet, suivant une déclaration de Murray, ils sont encore prisonniers sur le navire et on se propose de les envoyer en Angleterre ${ }^{39}$.

\section{Les directives de L'Évêque ET du Gouverneur}

Quant aux curés, quel a été leur rôle pendant les opérations de la conquête? Assumant la direction spirituelle d'une paroisse, quelle conduite ont-ils tenue lorsque l'ennemi s'est porté contre le territoire qu'ils avaient à desservir?

L'évêque avait donné là-dessus des directives précises: défense aux curés de porter les armes, chaque curé doit suivre sa paroisse

ancient father, d'un old friar que l'on se contente, à cause de son grand âge, de laisser chez lui avec une sentinelle: ce peut-être l'un ou l'autre de ces deux Pères, plutôt le $P$. Lauverjat que le P. Richer (alors curé de Lorette). Bien qu'on ne parle du retour des Jésuites au collège qu'en juin 1761 il est très probable que le vieux Père Lauverjat n'ait pas quitté la ville.

Knox parle d'un autre prêtre qui aurait été mêlé à un complot pour engager des soldats à déserter et qui aurait jugé plus prudent de se retirer du cóté de Montréal (Knox, Historical Journal, II: 280); il a sans doute confondu avec Charles Baudouin (c'est ainsi qu'il signe dans les registres de Notre-Dame-de-Québec), chassé à la même date par Murray pour avoir parlé de religion aux Anglais malades: voir le journal de Murray, cité dans Knox, Historical Journal, II: 278, n. 1).

35. Mémoire anonyme, dans Coll. Levis, IV: 191.

36. Vaudreuil à Lévis, 21 avril 1760, Coll. Lévis, VIII: 158.

37. Vaudreuil au Ministre, 22 juin 1760, APC, F 3, 16: 123.

38. Knox, Journal, II: 457s.

39. Discours de Murray, cité dans Knox, ibid., 474. 
"dans les profondeurs ou transmigrations"; si l'ennemi descend dans une paroisse et s'en rend maître, "le curé lui fera toutes les politesses possibles; le priera d'épargner le sang et les églises"; il faudra éviter "dans les prédications et même les conversations tout ce qui pourrait irriter le gouvernement nouveau"0". Et dans le même temps, le grand-vicaire Briand écrit aux curés de la Côte de Beaupré, la plus immédiatement menacée: "En conformité aux dispositions dont vous avez déjà été prévenu par l'ordre de M. le Gouverneur Général, vous vous retirerez dans les bois avec vos paroissiens, ayant eu soin d'emporter ce que vous pourrez des effets de votre église. Vous engagerez vos paroissiens à continuer dans leur retraite, tant que la flotte anglaise demeurera dans les environs de Québec ${ }^{41}$." Le gouverneur Vaudreuil, cependant, comptera en 1759 sur une collaboration plus utile des curés (ainsi que nous allons le voir bientôt) et, en 1760, en lançant Ja nouvelle campagne, il adresse une lettre circulaire aux curés du Gouvernement de Québec pour leur faire savoir que l'armée française marche sur Québec et il ajoute: "Je vous prie de vouloir bien employer le crédit, l'ascendant que votre caractère vous donne sur vos paroissiens pour leur faire connoitre qu'il est de leur religion, de leur honneur, de leur propre intérêt de se joindre tous avec armes et bagages à l'armée, et de surpasser même le zèle et l'ardeur des troupes, des Canadiens et des différentes nations sauvages qui la composent". Et il termine par cette exhortation pressante: "C'est maintenant, Monsieur, le moment où vous pouvez, en ce qui vous concerne, donner des preuves parfaites de votre zèle pour le service du Roi et de votre amour pour la patrie. Je saisirai avec plaisir cette occasion pour vous procurer des grâces de Sa Majesté42." C'était par cette lettre inviter les curés à faire intervenir dans le conflit le crédit et l'ascendant qu'ils avaient sur leurs paroissiens et le Gouverneur leur promettait des grâces royales en retour: c'était par conséquent amener les curés à sortir de leur rôle strictement spirituel et compromettre les relations du clergé avec les nouveaux maîtres.

40. Lettre circulaire de Mgr de Pontbriand, 5 juin 1759, Mandements, II: 1398.

41. Lettre circulaire, juillet 1759, Mandements, II: $140 \mathrm{~s}$.

42. Lettre circulaire écrite d MM. les curés nord et sud du gouvernement de Quebec, 16 avril 1760, Coll. Levis, IV: 221-223. 
Plusieurs curés ont joué un rôle assez actif pendant les opérations de la conquête; quelques-uns s'en tireront sans subir de conséquences, comme les curés de Baie-Saint-Paul, de Saint-Michel et Saint-Charles; un autre, le curé de l'Ange-Gardien, devra changer de Gouvernement; les curés de la Pointe-Lévy, de Charlesbourg et de Beauport seront emprisonnés; quant au curé de Saint-Joachim, il y perdra la vie de façon tragique.

\section{Trois amateurs en Défense militaire}

Louis Chaumont, curé de la Baie-Saint-Paul ${ }^{43}$, a soin, en juillet 1759 , d'avertir Vaudreuil "des mouvemens des navires" qui sont a sa portée et le Gouverneur, qui l'en remercie, ajoute: "Je compte fort sur l'exactitude de vos paroissiens à les observer. Je ne m'oppose pas qu'ils aillent attaquer les anglais qui sont sur l'Isle-auxCoudres... Je me flatte, monsieur, que vous voudriés bien exciter leur zèle \& leur persuader de plus en plus que c'est leur propre interet4." Le Gouverneur alla plus loin; comme un capitaine de milice de la Baie-Saint-Paul s'était mis au service des Anglais pour les guider, le Gouverneur enjoignit au curé Chaumont de faire arrêter et pendre sans cérémonie le traître Suisse; or cette dangereuse correspondance du Gouverneur français fut saisie par les Anglais parmi les papiers du curé Chaumont ${ }^{45}$. Cependant, il ne semble pas que les vainqueurs aient puni ni cherché à punir le curé de la BaieSaint-Paul pour le rôle qu'il venait de jouer dans la guerre.

Le curé de Saint-Michel-de-Bellechasse, Pierre Chaufour ${ }^{\mathbf{4 8}}$, prend quelque peu l'allure d'un capitaine de milice; à une lettre qu'il a écrite au Gouverneur pour le mettre au courant de son travail de défense, Vaudreuil répond: "Je ne puis que vous renouveller ma sensibilité à tous les soins que vous avés bien voulu vous donner pour engager vos paroissiens à se bien garder sur le fleuve" et Vau.

43. Louis Chaumonot de la Jaunière, qui aurait environ 59 ans (Tanguay, Répertoire, 110). Il signe simplement chaumont dans les registres de la paroisse sous le régime militaire.

44. Vaudreuil a Chaumont, 23 juillet 1759, Coll. Northcliffe, 229.

45. Knox, Historical Journal, II: 39.

46. Il n'avait alors que 35 ans; il mourra en juillet de l'année suivante (Tanguay, Répertoire, 122). 
dreuil envoie à Chaufour un ordre écrit (ordre que le curé avait lui-même demandé) "pour que ces habitans se portent avec le même zèle à suivre la garde particulièrement sur la concession du fleuvet7". Louis Sarault, curé de Saint-Charles-de-Bellechasset8, écrit lui aussi au Gouverneur pour rendre compte des mesures qu'il a adoptées et, en même temps, pour réclamer de ses paroissiens qui sont en service à Québec; le Gouverneur répond: "Il n'est rien de mieux que les justes mesures que vous avés engagé vos paroissiens à prendre pour se mettre à couvert des invasions de l'ennemi ${ }^{19}$." Ces trois curés ne semblent pas avoir été ennuyés par les nouveaux maîtres pour leur participation active, participation qui fut connue des Anglais, puisque les lettres que nous avons citées faisaient partie des papiers de Monckton.

\section{Le belliqueux Parent perd sa cure}

Le curé de l'Ange-Gardien, Joseph-Basile Parent ${ }^{50}$, fut plus actif que les précédents et les Anglais surent dès les débuts qu'ils avaient affaire à un adversaire. Au cours de l'été 1759, les Anglais mettent la main sur une lettre du gouverneur Vaudreuil à Parent, datée du 25 juillet, dans laquelle le Gouverneur lui mandait "entre autres choses, de lui faire part de tout ce qu'il pourroit apprendre d'extérieur, c'est-à-dire de lui servir d'espion"; les Anglais se plaignent de ce que ce curé "s'est trouvé avec une bande de paysans, sous un nommé Boucherville, à fusiller dans les bois, sur les troupes angloises ${ }^{51}$ ". Cette accusation est confirmée par un prisonnier canadien qui déclare aux Anglais que le curé se trouvait avec le parti de 200 Canadiens et sauvages que commandait Boucher-

47. Vaudreuil à Chaufour, 27 aout 1759, Coll. Northcliffe, 230.

48. Agé de seulement 33 ans (Tanguay, op. cit., 125). Il signe L. Sarault: fac.sim. dans La vieille église de St-Charles, 2.

49. Vaudreuil à Sarault, 28 aout 1759, Coll. Northcliffe, 230.

50. Il y avait alors trois curés Parent que l'on a souvent confondus. JosephBasile Parent, curé de l'Ange-Gardien, Louis-Laurent Parent, curé de Ste-Anne-deBeaupré, et Pierre-Clément Parent, vicaire à St-Vallier. Né à Beauport en 1724 (Maseicotte, Ste-Geneviève-de-Batiscan, 57), Joseph-Basile Parent n'avait donc alors que 35 ans.

51. Lettre écrite de la part des Anglois, Coll. Lévis, IV: 276-278. 
ville $^{52}$. Le 28 juillet, Vaudreuil parle de la visite que lui a faite la curé Parent: celui-ci lui a présenté des suggestions sur la composition d'un détachement et le Gouverneur recommande à Lévis de suivre ces suggestions ${ }^{53}$. Enfin, le 30 juillet, le curé Parent conduit aux quartiers généraux un homme "qui étoit chez les ennemis" lors de la "grande fusillade"

Le général Wolfe avait le droit, par conséquent, d'être mécontent du curé Parent; selon ce que rapporte Montcalm, "M. de Vaudreuil avoit écrit au sieur Parent, curé de l'Ange-Gardien, assez ridiculement suivant les apparences, et envoyé des couteaux à quelques Canadiens, qui font partie de l'équipement fourni par le Roi. Le général Wolfe a intercepté la lettre et en a fait faire une au dit curé d'un style relatif à son caractère bouillant et emportés." Les Anglais auraient même songé à s'emparer du curét.

Le curé Parent ne fut pas arrêté, mais il fut obligé de quitter la région après la capitulation de Québec: nous le retrouvons le 2 novembre 1759 à Yamaska, la paroisse la plus occidentale du Gouvernement des Trois-Rivières ${ }^{57}$. Son zèle intempestif et tout à fait déplacé lui avait fait perdre sa cure.

\section{On emprisonne Youville-Dufrost, puis on l'exile}

Le curé de la Pointe-Lévy, Charles Youville-Dufrostos, sera moins heureux que Parent: il connaitra l'emprisonnement. Par deux 1759).

52. Townshend Papers, dans Doughty, The Siege of Quebec, V: 253 (27 juillet

53. Vaudreuil à Lévis, 28 juillet 1759, Coll. Lêvis., VIII: 71s.

54. Montcalm à Lévis, 3'1 juillet 1759, ibid., VI: 209.

55. Journal de Montcalm, 12 août 1759, ibid., VII: 5888.

56. Lettre écrite de la part des Anglois, ibid., IV: 278.

57. Les historiens ont eu de la difficulté à suivre le curé Parent après son intervention dans la guerre: Casgrain écrit qu'il reste curé de l'Ange-Gardien jusqu'en octobre 1760 et qu'il sert ensuite de vicaire quelque part (L'Ange-Gardien, 197s.) Allaire écrit que Parent reste vicaire à l'Ange-Gardien jusqu'en 1763 et qu'il en part à cause de démêlés avec les autorités militaires (Dictionnaire, IV: 10s.) Le Canada ecclesiastique de 1913 (p. 134) le fait arriver aे Yamaska seulement en 1763. Il fallait tout simplement recourir aux registres d'Yamaska: on y constaté que Parent $\mathrm{y}$ signe régulièrement les registres à partir du 2 novembre 1759.

58. Charles-Madeleine Youville-Dufrost, fils de la veuve d'Youville, né en 1729 (Tanguay, Repertoire, 128). Connu souvent sous le nom de Dufrost, il signe cependant Ch. Youville Dufrost: voir le vol. III des registres de la Pointe-Lévy, conservés au presbytère de Lauzon. 
fois, le général Wolfe avait enjoint aux Canadiens de rester neutres dans le conflit et "de revenir avec leurs familles dans leurs habitatior s", moyerract quoi ils ne seraient point molestés ${ }^{59}$. Or, a la Poir te-Lévy et dars les enviro:s, il se pratiquait constamment une guerre d'escarmouches, à laquelle prenaient part surtout des Canadiers. Les Arglais résolurent à la fin de juillet 1759 de faire un nettoyage: cerer dart que le colorel Fraser se dirigeait du côté de Beaumo: $t$, le major Dallirg prit la direction de Saint-Henri; il y captura environ 300 personres, y compris le curé Youville-Dufrost et 54 hommes en état de porter les armes; tous les prisonniers furent conduits à bord de la flotte; le curé fut traité aimablement, mais resta prisorr rier ${ }^{00}$. Il n'était pas coutumier aux Anglais d'emprisonner les prêtres sa:s raison suffisante: on se souvient que le P. de la Brosse, capturé avec un groupe de femmes à la Pointe-aux-Trembles le 21 juillet, avait été relâché dès le lendemain, conformément au cartel qui assurait l'immunité aux aumôniers. Pourquoi alors retenait-on prisonnier le curé Youville-Dufrost? Lui qui se compromettra au cours de l'hiver 1759-1760, s'était-il compromis au cours de ce mois de juillet? Avait-il excité contre les Anglais les hommes qu'il avait avec lui en état de porter les armes? Le curé Youville-Dufrost n'avait que trente ans: l'ardeur de sa jeunesse l'avait-il porté à une participation active? Les Anglais qui avaient immédiatement libéré le $\mathrm{P}$. de la Brosse, devaient avoir leurs raisons sérieuses de détenir Youville-Dufrost. Il resta plusieurs semaines à bord du navire de guerre: capturé dars les derniers jours de juillet, il est encore sur le navire arglais le 4 septembre, lorsqu'il écrit à un officier anglais qui lui a fait passer des livres ${ }^{61}$. Combien de temps y reste-t-il encore? Il re dut être libéré qu'après la capitulation de Québec, le 18 septembre, peut-être seulement à la fin du mois ${ }^{62}$ : en tout cas, sa

59. Proclamation du 27 juin, Coll. Lévis, IV: 273-276; proclamation du 28 juin, dans Knox, Journal, I: 387-389.

60. Knox, op. cit., I: 440n.; Roy, Histoire de la seigneurie de Lauzon, II: 302s.; Ferland-Angers, Mère d'Youville, 154.

61. Lettre d'Youville-Dufrost, 4 septembre 1759, a bord de l'Orford, Coll. Northcliffe, 151.

62. Il n'y a aucune signature dans les registres entre le 18 juin et le 2 octobre (voir registres cités, plus haut, note 58 ). 
signature n'apparaît dans les registres de la Pointe-Lévy qu'à partir du 2 octobre.

Cependant, il n'en a pas pour longtemps à demeurer dans sa paroisse. Un conflit, dont on connait mal les détails, survient entre lui et le gouverneur Murray. Au cours de l'hiver 1759-1760, alors que les Français se montraient très actifs dans la région, allant même jusqu'à se fortifier dans l'église de la Pointe-Lévy ${ }^{63}$, le curé Youville-Dufrost aurait refusé de travailler au recensement de la paroisse et de faire fournir du bois, deux besognes que le gouverneur Murray lui avait demandées ${ }^{64}$. Les curés pouvaient se voir imposer le recenseme te leur paroisse, puisqu'ils tenaient les registres civils et dressaient même des contrats de mariage ${ }^{65}$; quant à la corvée du bois, il s'agissait là d'une mesure extraordinaire qui, ainsi que l'écrivait l'évêque, n'était pas de la compétence du cures6. Le curé Borel, de Sainte-Foy, qui s'était vu imposer ce recensement" et, très probablement, cette corvée de bois ${ }^{68}$, donna satisfaction au gouverneur Murray ${ }^{69}$. Le curé Youville-Dufrost, sans doute aigri par sa longue détention ou rendu plus indépendant à cause de la présence de troupes françaises dans sa paroisse, refusa de se conformer à la demande de Murray: le gouverneur s'en plaignit à un officier français qui rapporta la chose à l'évêque ${ }^{70}$. Mgr de Pontbriand jugea plus prudent d'éloigner le jeune curé et, en février

63. Les Anglais qui avaient occupé l'église au cours de l'été de 1759, l'évacuent après la prise de Québec, reviennent l'occuper en décembre 1759 , partent peu aprè et les Français les y remplacent; ces derniers en seront délogés le 13 février 1760 (Voir notre chapitre Les églises ont-elles souffert de la conquête?)

64. Pontbriand à Briand, 25 janvier 1760, AAQ, Evêques de Québec, I, 75: 3.

65. Trudel, Le régime militaire dans le Gouvernement des Trois-Rivières, 678.

66. Pontbriand à Briand, lettre citée.

67. Ordre au curé de Ste-Foy, 14 octobre 1759, Coll. Lévis, IV: 2788.

68. C'est ce que l'on peut déduire à la fois d'un ordre envoyé à ce même curé, le 21 octobre (ibid., IV: 279s.) et de l'importance que Knox accorde a Ste-Foy pour l'approvisionnement du bois (Journal, II: 276).

Le 7 novembre, Murray écrit qu'il a imposé 50 cordes de bois à chacune de trois paroisses des environs, pour l'Hôpital-Général: ce bois devait être payé par le roi. (cité dans Knox, Journal, II: 269n.)

69. Le 19 juin 1762, Murray lui donnera 25 livres anglaises (soit 600 livres françaises ou environ $\$ 600$.) pour réparer l'église que les Anglais avaient dynamitée en 1760 (ASQ, Comptes de depenses du Gouvernement de Quebec, 22).

70. Pontbriand à Briand, lettre citée, note 64 . 
1760, il annonce à son grand-vicaire que " $\mathrm{M}^{\mathrm{r}}$. Dufrost ne retournera plus a La pointe de Lévi"'1.

Youville-Dufrost quitte donc le Gouvernement de Québec pour éviter que la mésentente s'aggrave entre lui et le gouverneur Murray. Où va-t-il d'abord? Il semble bien que ce soit tout de suite chez son frère, Joseph-François Youville, curé de Saint-Ours: il y signe des registres en mars et avril $1760^{72}$. Le 13 juin, il est a Sainte-Rose, dans la paroisse la plus au nord du Gouvernement de Montréal: il y succède à l'ex-chanoine Lepage de Sainte-Claire qui se retire à Terrebonne $e^{73}$. C'est donc à Sainte-Rose qu'Youville-Dufrost apprend la capitulation générale du pays; il préfère $\mathbf{y}$ demeurer encore quelque temps: "je pense, écrit-il au grand-vicaire Briand, que vous ne trouverez pas mauvais que je reste dans le Gouvernement de Montréal. Côme Mr. Murray est toujours gouverneur de Québec, je crois quil ne seroit pas sur pour moi d'y retourner. jai exposé Ces raisons a $\mathrm{M}^{\mathbf{r}}$. Mongolfier qui m'a répondu que je pouvois rester a sainte rose que je dessert depuis plusieurs mois ${ }^{74}$." Il y reste donc jusqu'à la fin de l'été 1761 , puis, ne pouvant plus , contenir son ennui, il écrit à Briand qu'il est prêt à retourner à la "pointe lévi", pourvu "que vous vouiiez faire ma paix avec son excellence monsieur Murray. autrement je ne me say pas l'ame asser brave pour aller de moi même me présenter a québec ${ }^{75}$." Peu après, le grand-vicaire annonçait que la paix était faite et qu'YouvilleDufrost pouvait revenir dans sa paroisse où les habitants, écrit-il, le réclamaient "depuis deux ans qu'ils manquent de Secours ${ }^{76 "}$ ".

71. Pontbriand a Briand, 16 février 1760, AAQ, Evêques de Québec, I, 76: 1.

72. APJ-Sorel, 1756 a 1761 St Ours Copie des Registres: Youville-Dufrost signe des actes les 22 et 28 mars, 8 et 11 avril 1760 .

73. Le dernier acte de Lepage de Sainte-Claire dans les registres de Sainte-Rose est du 20 mai 1760 (APJ-M, Ste. Rose, 1745-1768). Dans un acte signé à Terrebonne, le 16 juin 1760, Lepage de Sainte-Claire s'intitule encore curé de Ste-Rose, mais c'est la dernière fois qu'il porte ce titre (APJ-St-Jérôme, 1759 a 1761 Terrebonne).

74. Youville-Dufrost a Briand, 18 septembre 1760 (lettre datée de Ste-Rose) AAQ, St-Joseph-de-Lévis, I: 19: 1. 6: 2 .

75. Le même au même, 18 septembre 1761 (lettre datée de Montréal), ibid., I,

76. Briand a Montgolfier, 26 septembre 1761, AAM carton I, 1. Les habitants avaient cependant été desservis assez régulièrement: par le récollet Didace Cliche, du 20 janvier au 8 février 1760; par Dosque, curé de Beaumont, du 4 mars au 27 
Le 12 octobre 1761, Youville-Dufrost signe son dernier acte a SainteRose; le 20 octobre, il est chez sa mère, directrice de l'Hôpital Général de Montréal, mais ce n'est que le 15 novembre qu'il reprend la signature des registres de la Pointe-Lévy"7.

Il n'avait pas rendu visite au gouverneur Murray en revenant dans la région de Québec; Murray s'en serait offusqué: "Une dame de Québec, écrit Youville-Dufrost à Briand, vient de me faire dire que s'étant trouvé à table cher $\mathbf{M}^{\mathbf{r}}$. Murray on à parlé de moi; et que son excellence à dit qu'il ne Croyoit pas que je fusse a la pointe lévi, et que s'il le sçavoit qu'il m'envoyeroit chercher pour m'apprendre a vivre. Si j'étois assuré que Cela fût vrai, je lui écrirois pour lui faire mes excuses. j'ai été deux fois cher lui sans lui pouvoir parler, et je n'ai eté que deux fois à québec depuis mon retour de montreal. si vous aver occasion de voir son excellence, je vous prie de vouloir faire ma seconde paix avec luir ${ }^{78}$." Briand lui recommanda de voir Murray à tout prix: "Il ne faut point dire qu'on vous a parlé que vous n'ayés pas vu $M^{\mathbf{x}}$ Murray, mais il faut venir au plus tôt et aller plusieurs fois dans le jour si vous ne le trouvés pas depuis 10 jusqu'à deux heures"9." Troublé dans sa retraite par les propos de cette dame que Murray avait reçue à sa table, YouvilleDufrost dut faire sa "seconde paix" avec le gouverneur: on n'entend plus parler d'incident entre l'un et l'autre. Après un emprisonnement de près de deux mois sur un navire de guerre et un exil d'un an et demi, Youville-Dufrost retrouvait enfin la tranquillité.

\section{D'AUTRES ARRESTATIONS?}

Le curé de la Pointe-Lévy ne fut pas le seul curé à se faire emprisonner pour un rôle trop actif dans le conflit: en juin 1760 Vaudreuil annonce que Murray détient sur un vaisseau de guerre

octobre 1760; par le récollet Daniel Normandin, du 22 octobre 1760 au 3 novembre 1761 (voir le vol. III des registres de la Pointe-Lévy, conservés au presbytère de Lauzon).

77. Voir registres cités, notes 73, 74, et APJ-M, Hôpital Général Sours Grises B.M.S., $1798-1795$.

78. Youville-Dufrost à Briand, 12 mars 1762, AAQ, St-Joseph-de-Levis, I, $13: 3$.

79. Briand a Youville-Dufrost, 14 mai 1762, AAQ, Copies de lettres, III: 107. 
les curés de Charlesbourg et de Beauport80. Le curé de Charlesbourg était alors Jean-Baptiste-Laurent Morisseaux, qui avait 40 ans environ $^{81}$; son emprisonnement aurait été assez long, si l'on en juge par son absence des registres, entre le 21 avril et le 11 octobre $1760^{82}$. Quant au curé de Beauport, Pierre-Simon Renauld, jeune prêtre de 29 ans $^{83}$, nous ne pouvons faire aucune supposition sur la durée de sa réclusion ${ }^{84}$. Qu'avaient-ils fait l'un et l'autre? Knox écrit au début de juin qu'un prêtre et deux ou trois jésuites ont été envoyés à bord des navires pour avoir "été trop empressés à encourager les Canadiens à la révolte ${ }^{85}$ ". Morisseaux ${ }^{86}$ et Renauld auraient-ils été arrêtés pour la même raison, après avoir pris trop au sérieux la circulaire que Vaudreuil avait adressée aux curés pour les inviter à soutenir la campagne de Lévis?

Le gouverneur Murray profita de cette arrestation de juin pour rappeler au clergé qu'il ne devait se mêler que du spirituel. Lorsque,

80. Vaudreuil au Ministre, 22 juin 1760, APC, F 3, 16: 123.

81. Tanguay, Repertoire, 119. Alors que son frère, curé de Champlain, signe Morrisseaux, le curé de Charlesbourg écrit Morisseaux (APJ-Q, Charlesbourg, 1759 dे 1760; 1760 d 1761).

82. Registres cités à la note précédente.

83. Tanguay, Répertoire, 129. Ce prêtre signe Renauld (APJ-Q, Beauport, 1759 d 1761).

84. Les actes des registres de Beauport, pour les mois de mai, juin et juillet 1760 sont tous signés par Renauld: ils se succèdent régulièrement, à intervalles très courts. Il est vrai que Renauld aurait pu les rédiger tous à une date ultérieure, en conservant l'ordre chronologique. Rien, en tout cas, ne nous permet de constater l'absence temporaire de Renauld, à part cette nouvelle de Vaudreuil; et l'on peut encore supposer que Vaudreuil s'est trompé; d'autant plus que les allusions contemporaines ne parlent que d'un prêtre emprisonné.

85. Knox, Journal, II: 4578.

86. Les relations de Morisseaux avec les Anglais, cependant, sont d'ordinaire excellentes. Après avoir obtenu pour son domestique une permission spéciale, il écrit au général: "J'apprend par la bouche de ce meme Domestique que dans quatre jours jay a vous faire ma reverence et vous presenter mon respect. Je dois monsieur par mil endroit a ce devoir et je leusse desja fais avec precipitation si quelqu'indisposition assez notable ne m'avois retenu" (Morisseaux à Monckton, 3 octobre 1759, Coll. Northcliffe, 283). Murray qui, en 1764, fait l'éloge de la grande charité de ce prêtre, lui avait concédé en $\mathbf{1 7 6 0}$ l'exploitation du poste de Saint-Augustin, dans le Labrador, pour lui permettre de subvenir aux besoins de deux jeunes enfants de d'Ailleboust de Cerry, enfants dont Morisseaux était devenu le "Dépositaire". Cette concession a dú se faire après la capitulation générale, de sorte que Murray n'aurait point gardé rancune à Morisseaux (voir là-dessus la lettre de Murray au Board of Trade, 25 août 1764, ainsi que le mémoire de Morisseaux, daté du 18 juillet 1764 et inclus dans la lettre de Murray: APC, C.0. 42, I, 2: 384-389). 
le mois suivant, des habitants de Sainte-Croix et de Lotbinière font par-devant lui leur soumission, accompagnés d'un prêtre ${ }^{87}$, Murray fait ure sortie contre le clergé qu'il accuse d'être "la source de tous les malheurs qui se sorit abattus sur les pauvres Canadiens qu'ils gardent dans l'ignorance et qu'ils excitent à la méchanceté et poussent à leur propre ruire. Vous avez sans doute entendu dire que j'ai pendu un capitaine de milice, que je détiens à bord d'un navire de guerre un prêtre et quelques jésuites qui doivent être envoyés en Grarde-Bretagre". Puis, interpellant directement le prêtre qui se trouvait là, il ajoute: Prêchez l'Évangile, qui seul est votre domaine; terez-vous en à vos devoirs et ne vous permettez pas, directement ni ir directement, de vous mêler d'affaires militaires ni de la querelle entre les deux Couronnes ${ }^{88}$ ". C'est, en somme, sur un ton plus violent, le corseil que l'évêque donnait à ses prêtres en juin 1759 .

Pour n'avoir pas manifesté assez de prudence, des prêtres avaient dû charger de cure ou même être emprisonnés pendant des semaines sur un navire de guerre. Pour deux membres du clergé, les conséquences de l'intervention seront tragiques: le curé de Saint-Joachim et l'ecclésiastique Joseph Couillard périront dans le conflit.

\section{Le curé Portneuf, victime de sa stratégie}

René Portneuf, de la grande famille des Robineau de Portneuf et de Bécancourt" ${ }^{89}$, âgé de 52 ans, était curé de Saint-Joachim lors de l'invasion anglaise. Comme les curés de Baie-Saint-Paul, de Saint-Michel et de Saint-Charles-de-Bellechasse, il intervient dans les opérations de la défense: dans le seul mois d'août 1759, il envoie trois lettres au gouverneur Vaudreuil pour l'instruire des mouvements des vaisseaux ennemis et pour lui décrire l'activité des habitants ${ }^{90}$. C'est l'époque où les Anglais font sur la côte de

87. Charles Dubergé était alors curé de Sainte-Croix; François Petit, celui de Lotbinière: nous ignorons lequel eut l'honneur de recevoir cette semonce de Murray...

88. Knox, Journal, II: 474. Murray, en remontant le fleuve, s'était arrêté sur 1a rive sud pour recevoir la soumission des habitants.

89. Né à Montréal en 1707, selon Tanguay (Répertoire, 108). Il signe René Portneuf, dans les registres de St-Joachim (copie microfilmée conservée à l'Institut généalogique Drouin). Sur sa famille, voir E.-J. Auclair, Les de Jordy de Cabanac, 187-217.

90. Vaudreuil à Portneuf, 20 aout 1759, Coll. Northcliffe, 229. 
Beaupré des incursions dévastatrices et, dans sa réponse du 20 août, Vaudreuil recommande au curé Portneuf que les habitants "se réunissent qu'ils soient toujours surveillans \& en état d'opposer la plus vive resistance aux anglais ${ }^{91}$ ". Dans cette résistance absolue, le curé Portneuf se mit à la tête de ses paroissiens.

A cause des versions discordantes, il est difficile de préciser les circonstances de la mort du curé Portneuf. Les Anglais incriminent totalement le curé. Selon Knox, qui situe l'affaire à Château-Richer et enregistre les faits avec quelque retard, Portneuf, avec environ quatre-vingts de ses paroissiens, s'était fortifié dans une maison imposante d'où il prétendait défier les troupes anglaises: "On va envoyer un détachement d'infanterie légère avec une pièce de campagne et un obusier pour les soumettre." Ce prêtre, continue la relation de Knox, envoya "un billet à l'un de nos officiers qui commande un poste dans les environs, le priant de lui faire l'honneur d'aller dîner avec lui, en compagnie de quelques-uns de ses lieutenants, l'assurant que lui-même et ceux qui seraient assez bons de l'accompagner s'en retourneraient en toute sûreté. Il ajoute que l'officier anglais se battant pour son roi et pour la gloire l'excusera, il l'espère, de se battre lui-même avec ses pauvres paroissiens pour la défense de leur pays. Notre officier a répondu qu'il regrette de ne pouvoir pas se rendre à cette invitation, mais qu'il espère dîner bientôt, dans le camp anglais, avec le galant prêtre et ses adhérents." Le lendemain, Knox écrit: "L'infortuné prêtre dont je parlais hier a été défait. Un détachement de troupes légères se mit en embuscade sur la lisière de la forêt près de la maison fortifiée. Aussitôt que la pièce de campagne eât commencé à fonctionner, le prêtre et ses hommes sortirent et ils tombèrent dans l'embuscade. Le prêtre lui-même et trente de ses compagnons ont été pris, tués et scalpés. On les a traités ainsi, parce qu'ils s'étaient déguisés en Indiens. La paroisse de Richet et la belle maison occupée par ce prêtre trop zélé sont maintenant en flammes"." Le capitaine Montrésor note de son côté: "Aujourd'hui a été tué et scalpé le prêtre qui commandait

91. Loc. cit.

92. Knox, Journal, II : 44s. Nous reproduisons ici la traduction donnée par E.-J. Auclair, dans Les de Jordy de Cabanac, 208. Knox enregistre le 24 aout l'envoi du billet de Portneuf, et le 25, sa mort, alors que le curé a été tué le 23 aout; c'est que Knox a probablement appris ces événements avec un retard do deux jours. 
un parti à Saint-Joachim, avec 20 de ses gens; ils n'opposèrent qu'une mauvaise résistance ${ }^{93 "}$ ". Un officier des Grenadiers de Louisbourg écrit: "Le 24, ils furent attaqués par un parti de Français, qui avait un prêtre pour commandant; mais notre parti en tua et scalpa 31, y compris le prêtre, leur commandant94." Enfin, le général Townshend note dans son journal à la date du 24 août: "Hier, 150 Canadiens avaient pris possession de quelques maisons près des bois. L'infanterie légère et les Rangers se disposèrent en cercle autour des bois et les entourèrent tous de très près; 30 d'entre eux furent tués et scalpés; parmi les scalpés, il y avait celui d'un prêtre ${ }^{95}$."

Aucun de ces quatre informateurs, Townshend, Knox, Montrésor et le sergent-major des Grenadiers, n'êtaient présents ${ }^{96}$ à l'affaire du 23 aout. Le récit de Knox est le plus détaillé, mais son témoignage reste indirect; et l'on peut laisser de côté cette histoire d'invitation à dîner envoyée par le curé Portneuf, parce qu'elle nous paraît assez peu vraisemblable; de même que cette accusation de déguisement que l'officier anglais porte contre les adversaires: Knox est le seul à la rapporter. Il est aussi le seul Anglais à situer l'affaire au Château-Richer, même si d'autres témoignages (celui de Montrésor et des témoignages français que nous verrons) situent la tragédie exactement à Saint-Joachim, on peut se demander si elle n'est pas survenue plutôt dans les environs de Sainte-Anne-deBeaupré, puisque c'est dans cette dernière paroisse qu'on a inhumé le curé Portneuf' ${ }^{97}$. Dans l'ensemble, la version anglaise se ramène à ceci : des Canadiens, dirigés par le curé de Saint-Joachim, opposent une résistance armée en se fortifiant dans une maison; les Anglais les font tomber dans une embuscade, en tuent une vingtaine ou une trentaine et les scalpent. C'est tout ce qu'on peut retenir de la version anglaise; on ne peut se fier aux détails qu'elle rapporte, parce que ces détails sont discordants.

93. Journal du capitaine John Montrésor, dans Doughty, Siege of Quebec, IV: 330 .

94. Journal d'un sergent-major des Grenadiers du général Hopson, ibid., V: 7.

95. Journal de Townshend, ibid., V: 261.

96. Au moment de l'affaire, ils semblent bien être tous les quatre au camp anglais de l'île d'Orléans.

97. Ste-Anne-de-Beaupré, Registre, II. 8 avril 1702 au 25 juil. 1708 - 2 Fét. 1727 au 26 Déc. 1785. 
Si l'on passe à la version française, on est aussi dérouté par la même discordance dans les détails. Une relation anonyme, qui n'est point celle d'un témoin direct, nous apprend que le curé de SaintJoachim et neuf de ses paroissiens ("ceux-cy avoient les armes à la main") furent capturés et maltraités, "mais le pauvre curé que les Anglois soupçonnèrent sans doute d'animer cette poignée de monde, le fut plus cruellement, puisque de sang froid ils luy firent couper le col au milieu de ses misérables habitants ${ }^{98}$ ". D'autres relations se montrent aussi réticentes sur l'activité militaire de Portneuf et insistent surtout sur la cruauté anglaise. Un journal, qui est probablement celui de l'aide-de-camp Marcel, porte ce qui suit: "Une vingtaine d'habitants de la Paroisse de Ste Anne ayant été surpris et emmenés prisonniers par un détachement des Ennemis furent tous massacrés inhumainement, et le Curé de cette Paroisse subit le même sort dans son Eglise même. Son corps fut indignement mutilé, on lui leva la chevelure, et son Eglise fut brâlée. Quelle barbarie! Les sauvages sont moins cruels, ils ne tuent presque jamais leurs prisonniers de sarg froid"9." Un déserteur anglais, qui a tout intérêt à parler comme les Français, annonce à Québec, selon le curé Récher, "qu'un détachement d'Anglais étant allé jusqu'à la quatrième église au-dessous de Beauport, y avaient trouvé douze hommes et entre autres un prêtre, qui se trouvant sans doute trop faible se jetèrent à geroux, demandant quartier, et que sans égard à leurs prières, les Arglais avaient tiré et tué le prêtre avec quelques autres; cela fait craindre pour M. de Portneuf, curé de St-Joachim"; puis, ayant appris de nouveaux détails par une autre source, le curé Récher ajoute: "C'est lui en effet qu'ils ont pris avec 8 habitants, après les avoir attirés du bois dans la plaire, en feignant de fuir et les avoir environnés. Ils lui orit levé la chevelure et fendu la tête. Il y a lieu de penser qu'ils ne l'ont pas tué à coups de fusil, mais à coups de sabre, ainsi que 7 habitants dont les cadavres ont été trouvés dans la maison avec celui de M. de Portneuf. Les Anglais étaient piqués contre nos gens qui les avaient injuriés de loin"'. $\mathrm{V}: 321$.

98. Relation anonyme du siège de Québec, dans Doughty, Siege of Quebec,

99. Journal abrége d'un aide-de-camp, ibid., V: 293.

1. Journal du curé Récher, dans BRH, IX (1903), 372. 
Même réticence et même ton dans la lettre de Bígot à Lévis: "J'oubliois d'avoir l'honneur de vous dire que les Anglois ont tué l'abbé Portneuf, curé de Saint-Joachim, et reuf habitants avec lui, quoiqu'ils se fussent rendus prisonniers. Ils étoient dix habita. ts avec lui; un seul s'est sauvé. Ils furent cernés dans un bois par deux certs rangers. Le curé a eu la tête ouverte en quatre et toute la chevelure faite. M. Wolfe est cruel'." Montcalm est le seul cortemporain français à écrire immédiatemert, qui parle de "résistan ce"; il écrit dans son journal, le 31 août 1759: "On rous a débité, il y a quelques jours, qu'un détachement des enremis, destiré à brûler la paroisse de Saint-Joachim, avoit éprouvé quelque résistance de la part d'une cinquantaire d'hommes corduits rar leur curé, le sieur de Portreuf. N'ayant pas été les plus forts, huit hommes ort été tués et la chevelure levée. Ils ont été si mutilés qu'on a eu pei: e à reconnoître le cadavre de l'ecclésiastique. Ce fait s'est vérifiés." E: ifin, nous devons enregistrer ici la relation du sieur de Courville, bien qu'elle ait été écrite après la conquête et que l'auteur se mo: tre assez souvent anticlérical'; le sieur de Courville, gé éralement bie:? re: seigné, corrobore la version anglaise: "Quelque tems après fut tué l'Abbé de Portneuf, Curé de la paroisse de St. Joachim; ce prêtre avait endossé la cuirasse dès l'entrée des Anglois dars la rivière; il étoit constamment à harceler l'armée à la tête des habita: ts de sa paroisse; un détachemert, qui s'étoit trouvé la veille à portée de cette paroisse, avoit été ir sulté de paroles par les habitants qui avoient à leur tête ce prêtre; c'est ce qui fit que le lendemain ce détacheme. $t$ revint vers St. Joachim, avec ordre de les attaquer, ce qu'il fit; L'Abbé Portneuf fut fait prisonnier avec huit habitants; les Anglois le tuèrent, et reprochère:it au prêtre d'être sorti de so: état, et d'avoir excité ces habitants à les insulter"s". Un autre mémoi"e qu'on a attribué à ce même sieur de Courville et qui n'est probableme t pas de lui ${ }^{6}$, donne à peu près la même version: "L'abbé de Portneuf,

2. Bigot à Lévis, 1er septembre 1759, Coll. Lévis, IX: 53.

3. Journal de Montcalm, Coll. Lévis, VII: 600s.

4. Sur le sieur de Courville, voir A. Fauteux, Le S... de C... enfin démasqué, dans les Cahiers des Dix, V (1940): 231-292.

5. Mémoires du S... de C... (éd. de 1873), 157.

6. Là-dessus, voir Fauteux, op. cit., dans les Cahiers des Dix, V (1940):268-273. 
d'une famille du Canada, avait été envoyé comme curé dans une paroisse nommée St-Joachim, derrière le camp anglais du Sault Montmorency. Ce prêtre s'était retiré dans les profondeurs des terres de sa paroisse, d'où sortant de temps à autre à la tête de quelques-uns de ses paroissiens, il faisait des courses sur l'ennemi. Le général Wolfe lui avait écrit plusieurs fois à ce sujet, mais rien n'avait pu contenir l'humeur guerrière de ce curé. Enfin il eut le malheur de tomber un jour entre les mains d'un parti anglais, qui l'hachèrent en pièces avec ses gens ${ }^{7}$."

Pas plus que du côté anglais, nous ne trouvons ici de témoignages directs; il y a bien cet unique habitant qui, selon Bigot, s'est sauvé, mais ce survivant n'a fait aucune déposition formelle. De ceux qui notent les faits au jour le jour, Montcalm est le seul à parler de la résistance de ces paroissiens, conduits par le curé Portneuf. Quant aux Mémoires de Courville et au Mémoire du Canada, rédigés peu après les événements, ils rappellent sans ambage les courses faites sur l'ennemi par le curé et son ardeur guerrière. Nous avons tout de même un document qui donne un témoignage immédiat et que l'on doit accepter, témoignage qui rejoint la version de Montcalm et de Townshend; c'est l'acte d'inhumation, rédigé par le curé Louis-Laurent Parent. On lit en effet dans les registres de Sainte-Anne-de-Beaupré, à la date du 26 août 1759, qu'on a inhumé René Portneuf "qui a été massacré par les anglois le 23 de ce mois etant a la tete de sa paroisse pour la deffendre des incursions et hostilités quy faisoit lennemis.8."

Cet acte d'inhumation, rédigé trois jours après par un curé qui se trouvait près de l'endroit où s'est produite la tragédie, confirme les témoignages indirects des Anglais, de Montcalm et du sieur de Courville sur l'activité militaire du curé Portneuf. Nous ne pouvons plus en douter: le curé était à la tête de sa paroisse "pour la deffendre des incursions et hostilités quy faisoit lennemis". Lorsqu'il est tombé entre les mains des Anglais, ceux-ci l'ont châtié avec la cruauté coutumière à cette époque. Il est ridicule d'écrire, comme le fait le curé Récher (sans doute mal renseigné), que les Angla!̣ "étaient piqués contre nos gens qui les avaient injuriés de loin"; la faute des

7. Mémoire du Canada, dans RAPQ (1924-1925), 164.

8. Registres cités, voir plus haut note 97 . 
paroissiens et du curé était plus grave: le général Wolfe avait demandé aux habitants de rester neutres dans le conflit et c'est pour les punir de leur "petite guerre" qu'il avait envoyé des partis dévaster la côte de Beaupré. En s'opposant à ces partis, les paroissiens de Saint-Joachim défendaient leurs biens, mais se trouvaient en même temps sans aucun status de soldats réguliers: ils n'étaient point protégés par le cartel de février 1759 ni par la coutume militaire. Les Anglais, aigris sans doute par les courses harassantes faites sur eux par le curé Portneuf, donnèrent libre cours à leur rancœur.

Les prêtres qui accompagnaient les troupes avaient droit à l'immunité, à condition évidemment de ne point se comporter en militaires: or ni Montcalm, ni Vaudreuil, ni surtout l'évêque ne présentèrent de protestations contre le massacre du curé Portneuf. Pourtant, si le curé eât été innocent de toute activité militaire, comme le prétend Auguste Gosselin', quelle occasion magnifique on eât trouvé là pour accabler les Anglais, pour stigmatiser la conduite des généraux anglais, pour crier à la persécution religieuse! Quel magnifique complément à l'affaire Jumonville! L'évêque n'a rien dit, Vaudreuil n'a point protesté. On n'a rien fait, parce qu'il n'y avait rien à faire: le curé Portneuf, en conduisant ses gens dans cette petite guerre (même s'il ne portait pas d'arme), agissait d'une façon irrégulière. En défendant sa paroisse, il se montrait patriote et chevaleresque, si l'on veut, mais il compromettait gravement la cause de l'Église; il se compromettait surtout lui-même: il a payé de sa vie son zèle intempestif.

\section{L'ecclésiastique Couillard, TuÉ DaNs UNe escarmouche}

Enfin, un autre membre du clergé périt dans le conflit: l'ecclésiastique Joseph Couillard, fils de Jean-Baptiste Coulllard des Écores,

9. Gosselin écrit que Portneuf n'était pas "armé lorsqu'il fut pris et massacré par les Anglais"; nous l'admettons parce que rien ne prouve le contraire; mais Gosselin ajoute: "Il s'était retiré dans les bois avec ses paroissiens, conformément à l'ordonnance de l'évêque"; or un jour que les habitants sortent du bois pour donner la chasse aux Anglais, Portneuf les accompagne "pour leur administrer, au besoin, les secours de son ministère"; il est cerné et massacré (Gosselin, L'Eglise du Canada, III: 511). C'est résumer très pauvrement l'activité militaire du curé Portneuf, dans une vaine tentative pour en faire une victime innocente. 
D

eigneur de la Rivière-du-Sud ${ }^{10}$. Torsuré et clerc-minorétil, Joseph Couillard n'avait pas le droit de porter les armes, mais il les porta quand même. Agé de seulement 21 ans, se laissant sans doute emporter par l'ardeur de sa jeunesse et agissar.t comme un bon nombre des étudiants du Séminaire de Québec, il se mit au service de l'armée française. Le 9 août 1759, on apprend qu'il est blessé et en même temps qu'il mérite les éloges de ses supérieurs; Vaudreuil écrit à Bougainville: "Je souhaite que la blessure de l'abbé Couillard soit légère. Il est bon gentilhomme et si ce n'etoit qu'un torsuré et qu'il préféra le service, il seroit bien fait pour y être placé12"'. Sa participation militaire était assez brillante pour être remarquée par Vaudreuil. Nous ignorons si l'ecclésiastique Couillard se remit assez tôt de sa blessure pour reprendre le service. Il semble bien re quitter Québec qu'après la défaite des Hauteurs d'Abraham; le 14 septembre, il était en route pour Saint-Thomas-de-Mortmagry avec quelques compagnons. Comme on arrivait en vue du village, la ruit tombée, on fit la rencontre d'ure petite troure d'Arglais: on er gagea le combat, l'ecclésiastique Couillard fut tué avec Jean-Baptiste Couillard, seigneur primitif ${ }^{13}$. Comme le curé de Sairt-Joachim, cet ecclésiastique est mort pour sa patrie, bien qu'il re fut pas conforme à son état de prendre part au conflit armé: par son devoir

10. Abbé A. Couillard-Després, Histoire des seigneurs de la Rivière du Sud et de leurs allies Canadiens et Acadions (Saint-Hyacinthe), 1912, 283-284.

11. Dans la liste des boursiers du Séminaire de Québec, en 1758-1759, on trouve deux Couillard: "Couillar maj" et "Couillar min" (ASQ, Application des Fondations. De 1730 d 1852, 17). Le premier est l'ecclésiastique Joseph, âgé de 21 ans; l'autre est son frère, André, âgé de 19 ans (Couillard-Després, op. cit., 205s.) Ce dernier reviendra au Séminaire où il sera boursier en 1762-1763 et en 1763-1764, mais ne se rendra pas à la prêtrise. IV: 19

12. Vaudreuil a Bougainville, 9 aout 1759, dans Doughty, Siege of Quebec,

13. Voir là-dessus APJ-Montmagny, St-Thomas, 1690-1703, 1798- 99,1759 1763: acte d'inhumation, 22 septembre 1759; Casault, Montmogn.y, 84s.; Tanguay, $A$ travers les registres, 174; Couillard-Després, op. cit., 283s.; Gosselin, L'Egliee du Canada, III: 517, n. 42. Casault rapporte aussi un récit traditionnel, assez différent de celui des historiens; selon ce récit, le seigneur revenait de Québec avec des compagnons; à quelqu'un qui lui aurait fait remarquer le danger de voyager la nuit a cause des Anglais, le seigneur aurait répondu qu'il avait une passe; peu après, il fut atteint par des balles anglaises. L'histoire de cette passe est assez invraisemblable puisqu'il n'y avait encore aucune paix de conclue (voir ce récit, dans Casault, $o p$. cit., $428 \mathrm{~s})$. 
comme en vertu des canons de l'Église, il était obligé de se tenir hors de ce conflit.

\section{Conclusion}

Certains membres du clergé ont donc pris une part active au conflit, se laissant emporter par leur patriotisme et oubliant que leur rôle devait se borner strictement au ministère des âmes. Pour quelques-uns, la participation au conflit reste assez anodine: les jésuites Gounon et Roubaud, les curés Chaumont, Chaufour et Sarault servent d'informateurs auprès des autorités militaires; d'autres, et c'est le cas des sulpiciens de Terlaye et Picquet, dépassent de beaucoup leurs simples fonctions de missionnaires. Un jésuite est arrêté pour avoir caché sous terre des munitions, après la prise de Québec: mais on n'a pas là-dessus des données absolument certaines. Un autre jésuite, le Père de la Brosse, est fait prisonnier à la Pointe-aux-Trembles, mais comme les Anglais n'ont rien à reprocher à cet aumônier, il est immédiatement relâché. D'autres prêtres s'en tirent moins facilement: le curé Joseph-Basile Parent, de l'AngeGardien, est obligé d'abandonner sa cure et d'aller se réfugier dans la paroisse la plus occidentale du Gouvernement des Trois-Rivières; le sulpicien Picquet juge préférable de s'enfuir par la Louisiane; le curé Youville-Dufrost, pour avoir joué un rôle qu'on ne peut très bien préciser mais qui fut trouvé condamnable par les Anglais, reste plusieurs semaines prisonnier à bord d'un navire de guerre, puis, parce qu'il ne montre pas suffisamment de souplesse à l'égard de Murray, il doit aller se réfugier pendant un an et demi dans le Gouvernement de Montréal. Les curés de Charlesbourg et de Beauport, ainsi que des jésuites non identifiés, auraient connu eux aussi l'emprisonnement pour avoir probablement excité les Canadiens à la révolte. Il périt deux membres du clergé qui sont intervenus très activement dans le conflit: l'ecclésiastique Couillard, qui sert d'une façon excellente sous Bougainville, se fait tuer en retournant chez lui après la défaite de Montcalm; René Portneuf, curé de SaintJoachim, se fait massacrer à la tête de ses paroissiens dont il dirigeait la résistance.

Ce ne sont là que des cas isolés; on doit même dire que ce sont des exceptions. La très grande majorité du clergé s'est tenue en 
dehors du conflit armé: elle se conformait ainsi aux instructions précises de l'évêque. Alors que le gouverneur Vaudreuil manquait à la prudence en espérant faire servir les curés à ses fins militaires, le gouverneur Murray exigeait que le clergé s'en tînt exclusivement à son ministère sacerdotal; les prêtres qui, dans ces circonstances difficiles, se sont laissé conduire par les simples règles de la prudence, n'ont point subi de dérangement de la part des vainqueurs: ces prêtres ont constitué la presque totalité et il faut s'en souvenir pour comprendre les relations du clergé avec les nouveaux maitres sous le régime militaire.

\author{
Marcel TRudel, \\ professeur d'Histoire du Canada \\ $\grave{a}$ l'Université Laval.
}

Perrin - Dulac (Voir notre no de septembre 1953, article de M. Fernand Grenier).

Biographie universelle, ancienne et moderne, (Paris, L.-G. Michaud, 1844), Tome 76 (Supplément), p. 495. PERRIN-DULAC, (F.-M.) - litterateur et géographe, fut d'abord sous-prefet de Sancerre lorsqu'il revint de ses longs voyages, et le fut ensuite de Rambouillet, où il mourut on juillet 1824. Il avait publie:

I - Voyage dans les deux Louisianes et chez les nations sauvages du Missouri, par les États-Unis, l'Ohio et les provinees qui le bordent, en 1801, 1802 et 1803, avec un Aperçu des mœurs, des usages, du caractère et des coutumes religieuses et civiles des peuples de ces diverses contrées. Paris, 1805, in-8o, avee pl. et cartes.

II - Salomon, poème traduit de l'anglais de Prior (voyez ce mot) XXXVI, p. 110) Paris, 1808, in-80.

(Note transmise par le Père Paul Desjardins, s.j., Collège Sainte-Marie, Montréal). 